[2] R. J. Bryant, A. C. Chamberlain, A. Morgan, G. S. Spicer. Radiostrontium fallout in Biological Materials in Britain AERE/HP/R 1056. Septembre 1956.

[3] G. M. Milton, W. E. Grummits. Ion Exchange Methods for the Quantitative Separation of the Alkaline Earths, and their application to the determination of ${ }^{90} \mathrm{Sr}$ in Milk Ash. Canad. J. Chem., 1957, 35, 541-551.

[4] A. Miserez. Le strontium 90, son identification et son dosage spécialement dans le lait. Mitt. Geb. Lebensmitteluntersuchung und Hygiene. I. $1957,48,6,468-476$; II. $1958,49,1,36-60$.

[5] L. Jeanmaire, H. Jammet. Service d'Hygiène Atomique et de Radiopathologie. Analyses radiotoxicologiques urinaires (Pas encore publié).

\title{
RECHERCHES ÉLECTROPHORÉTIQUES SUR QUELQUES FROMAGES ITALIENS
}

\author{
par \\ SERGIO ANNIBALDI
}

Il n'y a aucun doute que l'électrophorèse a apporté une contribution fondamentale à l'étude de la structure protéique des fromages et des transformations qu'elle subit pendant la période de maturation ; un phénomène qui, comme on le sait bien, a lieu d'une façon prépondérante à la charge de la partie protéique qui est plus ou moins protéolysée.

Des études faites jusqu'à présent, par plusieurs auteurs [1] [2] [3] il est ressorti que les fromages ont une physionomie électrophorétique à eux, une physionomie qui, tout en répétant l'aspect de la $p$-caséine, s'éloigne plus ou moins de son schéma fondamental, en ce qui concerne soit le nombre des fractions électrophorétiques présentes, soit le rapport quantitatif où ces fractions se trouvent entre elles. Ces différents caractères dépendent des mêmes causes qui rendent différents les fromages mêmes à la suite d'autres propriétés (caractères organoleptiques), telles probablement la technique de travail et le degré de protéolyse qu'ils ont atteint.

Si l'on examine l'électrophorétogramme d'un fromage, après avoir gardé ce dernier dans l'eau, haché menu, pendant quelques heures, on peut remarquer comme il est différent de l'électrophorétogramme obtenu du fromage non traité à l'eau. Cela signifie qu'au moyen de l'extraction aqueuse on a privé le fromage des substances azotées solubles, qui sont naturellement restées délayées dans l'eau, ce qu'on peut aisément vérifier en observant l'aspect électrophorétique du liquide préparé à propos. L'examen du résidu insoluble en eau d'un fromage peut donc fournir une vision suffi- 
samment approximative du degré et du type de maturation subie en observant quelles fractions caséiniques, et en quelle quantité, se sont dissoutes dans l'eau, par rapport au même produit non macéré.

Le sujet de l'examen électrophorétique des fromages se présente donc intéressant, non seulement parce qu'il réserve certainement d'utiles remarques, mais encore parce que le nombre des produits examinés est jusqu'ici très faible. Des nombreux fromages italiens, on n'a, actuellement, observé que le Parmesan [5]. C'est justement pour contribuer à cette étude qu'on a fait ce travail, portant sur l'examen du comportement électrophorétique de fromages italiens, dont quelques-uns typiques et bien connus, choisis de façon à comprendre des produits différents soit pour la technique de travail, soit pour le procédé de maturation. On a effectué, au moyen de l'examen du résidu insoluble en eau, l'étude de leurs caractéristiques de maturation.

Les fromages pris en considération sont groupés dans le relevé ci-dessous :

\begin{tabular}{|c|c|c|}
\hline Fromages erus & $\begin{array}{l}\text { à maturation rapide } \\
\text { (dans } 30 \text { jours) }\end{array}$ & $\begin{array}{l}\text { Italique } \\
\qquad 20-30 \text { jours }\end{array}$ \\
\hline Temp. $35^{\circ} \mathrm{C}$. & $\begin{array}{l}\text { à maturation moyenne } \\
\text { (dans } 180 \text { jours) }\end{array}$ & $\begin{array}{r}\text { Gorgonzola } \\
120 \text { jours }\end{array}$ \\
\hline Fromages à pâte filée & $\begin{array}{l}\text { à maturation rapide } \\
\text { à maturation moyenne }\end{array}$ & $\begin{array}{l}\text { Mozzarella } \\
\text { heures } \\
\text { Provolone } \\
\text { 3-6 mois }\end{array}$ \\
\hline
\end{tabular}

Fromages demi cuits
Temp. $35-48^{\circ} \mathrm{C}$.$\left\{\begin{array}{cc}\text { à maturation moyenne } & \text { Fontine vallée d'Aoste } \\ \text { à maturation lente } & 3-4 \text { mois } \\ \text { (de } 180 \text { jours à } 3 \text { ans }) & \text { Fromage de brebis romain } \\ 8-9 \text { mois }\end{array}\right.$

Fromages cuits
Temp. $48-56^{\circ} \mathrm{C}$.$\quad\left\{\begin{array}{cc}\text { à maturation lente } & \text { Fromage Parmesan } \\ 2-3 \mathrm{ans}\end{array}\right.$

Pour mieux fixer les caractéristiques de ces produits, il faut en toucher quelques mots, en ce qui concerne leur technologie.

Italique. - Fromage cru produit en meules, du poids de 2 kilogrammes, hautes de 5 centimètres, avec un diamètre de 20 centimètres. On le produit avec du lait de vache frais, généralement pasteurisé, qu'on fait cailler lentement, à $35-36^{\circ} \mathrm{C}$. Le caillé, haché aux dimensions d'une noisette, après une courte période de purgation, est mis en moules et conservé à $20^{\circ} \mathrm{C}$. en lieux très humides pendant 2 à 3 jours. La salaison est à sec et la maturation continue 
pendant 20 jours environ, dans des magasins très humides, à 5-6 ${ }^{\circ} \mathrm{C}$. de température.

Gorgonzola. - C'est un fromage à pâte bleue, produit avec du lait de vache entier. Il a une forme cylindrique du poids de 10 à 12 kilogrammes, haute de 20 centimètres et avec un diamètre de 22-28 centimètres. A l'intérieur la pâte présente des rayures bleuverdâtre caractéristiques, dues au Penicillium Weidmanni West var. fuscum, qu'on ajoute au lait pendant qu'on le travaille ou qu'on fait développer en employant des procédés appropriés. Le lait est caillé à $35^{\circ} \mathrm{C}$. en 20 minutes environ et le caillé, haché aux dimensions d'une grosse noix, après une courte période de purgation, est recueilli dans des toiles; ensuite, on le place dans les bandeaux, où il reste pendant 3 à 4 jours, à $20^{\circ} \mathrm{C}$. La salaison est à sec. La maturation dure au total entre dessèchement et affinement, 4 à 5 mois, à une température de 5-6 $6^{\circ} \mathrm{C}$.

Mozzarella. - Ce fromage, produit à l'origine avec du lait de buffle, on l'obtient maintenant avec du lait de vache aussi. Il a une forme ovoïdale et un poids de 300 grammes environ. On le produit avec du lait de vache, qu'on fait cailler en 30-60 minutes à la température de $30-35^{\circ} \mathrm{C}$. On réduit le caillé aux dimensions d'une noisette, et, après une période de purgation, on le laisse mûrir jusqu'à ce qu'il acquière des propriétés filantes. On coupe alors la pâte en bandes très minces et avec de l'eau chaude à la température de $70-80^{\circ} \mathrm{C}$., on la manipule et on la forme convenablement. La salaison au moyen de saumure à, $10 \%$ dure deux heures, après quoi, le produit est prêt à la consommation.

Provolone. - C'est un fromage cru de pâte filée, produit avec du lait de vache entier. Il a une forme d'ordinaire à trone de cône et un poids qui varie entre 3 et 6 kilogrammes. Le lait est caillé à $36-37^{\circ} \mathrm{C}$., en 8-10 minutes, avec de la présure en pâte de chevreau très piquante. Le caillé, réduit à des grumeaux comme des noisettes, est soumis à une période de purgation en réchauffant légèrement le petit-lait et puis on l'abandonne pour qu'il se recueille à former comme une galette sur le fond de la cuve en bois, dans laquelle a lieu le travail. On laisse fermenter la galette et lorsque, par effet de décalcification, elle a atteint un certain degré de plasticité et d'élasticité, on la coupe en bandes minces et on la plonge en eau chaude à $80-90^{\circ} \mathrm{C}$., où, au moyen d'une manipulation convenable, elle est filée et façonnée d'abord à la main, ensuite en coules appropriées. La salaison a lieu avec de la saumure légère. La maturation dure 3-6 mois selon le type. Ce fromage peut aussi être fumé.

Fontine de la vallée d'Aoste. - On produit ce fromage avec du lait de vache entier d'une traite; il a une forme cylindrique légè- 
rement concave, haute de $8-10$ centimètres et un diamètre de $35-45$ centimètres. Une forme pèse de 8 à 18 kilogrammes. Le lait est caillé en 30-40 minutes à la température de 32-340 C., au moyen de présure naturelle, préparée par le fromager même. Le caillé, brisé en petits grumeaux des dimensions de grains de blé, est cuit à $45-48^{\circ} \mathrm{C}$. Dès qu'on a atteint cette température, le caillé est gardé en mouvement pendant 30-40 minutes jusqu'à ce que les grains soient bien secs. La masse caséeuse unique qui s'est formée dans la chaudière est placée dans les bandeaux et comprimée au moyen $\mathrm{du}$ pressoir. La salaison est à sec; la maturation dure 3.4 mois.

Fromage de brebis romain. - Ce fromage est produit avec du lait de brebis entier. Il a une forme eylindrique haute de $15-22$ centimètres, dont le poids est de 10-12 kilogrammes environ. Le lait de brebis frais est chauffé à $37-38^{\circ} \mathrm{C}$. et caillé au moyen de présure naturelle d'agneau préparée par le fromager lui-même. Le caillé, réduit en grumeaux des dimensions de grains de blé, est porté au point de cuisson, ce qui consiste à le chauffer jusqu'à $43-44^{\circ} \mathrm{C}$. On laisse déposer ces grumeaux dans le fond de la chaudière, où le fromager, en présence du petit-lait, manipule la masse caséeuse, qui est répartie et placée dans des meules, où, à l'aide d'opérations manuelles convenables, on favorise la sortie du petit-lait. La salaison est à sec et dure 3-4 mois; la maturation dure 8-9 mois.

Fromage Parmesan. - On le produit avec du lait de vache demi-écrémé de deux traites. Il a une forme cylindrique haute de $22-23$ centimètres, un diamètre de $30-45$ centimètres, et un poids moyen de 30-35 kilogrammes. Le lait de deux traites, en partie légèrement tourné, est mélangé dans la chaudière et, après l'adjonction préalable d'une quantité calculée de sérum-ferment, il est caillé à la température de $32-33^{\circ}$ au moyen de présure de veau. Le caillé est réduit en grumeaux des dimensions d'un grain de blé et, à l'aide de la chaleur, convenablement dosée, épuré du petitlait. Enfin, les grumeaux bien secs sont cuits en élevant la température à 54-56 $\mathrm{C}$. La masse caséeuse unique qui se dépose sur le fond des chaudières caractéristiques est placée dans des bandeaux et légèrement comprimée. La salaison a lieu à l'aide de saumure généralement saturée. La maturation dure 2-3 étés.

\section{Partie expérimentale}

L'outillage employé pour ce travail est constitué d'un appareil pour électrophorèses à phase libre HILGER et WATTS de Londres, pourvu d'un système optique Schlineren Philpot pour l'absorption de l'ultra-violet. La lampe à vapeurs de mereure produit une lumière qui, filtrée au moyen du filtre jaune, prend une longueur 
d'onde de $546 \mathrm{~m} /$ microns qui fournit des gradients mesurables même avec de forts délayages.

Dans la détermination électrophorétique on a employé un courant de $12 \mathrm{~mA}$ à la température d'expérience de $+0^{\circ} 5 \mathrm{C}$. $( \pm 0,01)$. Les photos ont été prises après 90 minutes d'électrophorèse.

On a aussi utilisé, pour rendre suffisamment transparentes les solutions lactescentes des fromages, une ultra-centrifuge préparatoire réfrigérée Spinco Mod. L.

La solution tampon employée a un $p \mathrm{H}$ de 8,6, une force ionique de 0,1 et est composée de grammes $2,8 \%$ d'acide diétilbarbiturique (véronal Bayer) et de grammes $20,6 \%$ de diétilbarbiturate sodique (véronal sodique Bayer) en eau distillée.

Les fromages soumis à l'épreuve avaient atteint le degré de maturation et tous leurs caractères particuliers.

\section{Préparation des échantillons totaux}

10 ou 20 grammes de fromage, selon leur contenu aqueux, broyés menu ou râpés, sont dissous à $40^{\circ} \mathrm{C}$. en $100 \mathrm{~cm}^{3}$ d'une solution à $10 \%$ de sodium citrate neutre RP Erba.

Le fromage est convenablement agité jusqu'à dissolution. Par une première centrifugation à la vitesse de 1.500 tours/min. on sépare les particules non dissoutes et la graisse affleurant dans les tuyaux de la centrifuge. La caséine est ensuite coagulée par adjonction d'acide acétique à $10 \%$ et recueillie par centrifugation. Le sédiment est suspendu en eau et lavé, ensuite nouvellement sédimenté et enfin dissous en $40-50 \mathrm{~cm}^{3}$ de solution tampon à la température de $40-50^{\circ} \mathrm{C}$. La solution fortement lactescente est centrifugée au moyen de l'ultra-centrifuge préparatoire pendant 10 minutes à la vitesse de $\mathbf{4 0 . 0 0 0}$ tours/min.; elle devient ainsi parfaitement limpide. Cette centrifugation rapide n'engendre pas des modifications importantes dans les électrophorétogrammes. Le liquide ainsi obtenu est dialysé, dans un tuyau de cellulose Visking, contre la solution tampon, pendant 63-65 heures à la température de $+4^{\circ} \mathrm{C}$. Pendant la dialyse on a une part d'azote sensible, qui, en certains cas, atteint 25-30\%. Avant de soumettre la solution à l'électrophorèse, il faut déterminer le contenu en azote, de façon à en porter la concentration protéique entre 1,2 et 1,8\%, au moyen de dilution avec solution tampon.

\section{Préparation des échantillons de la fraction insoluble}

10 à 20 grammes de fromage râpé ou haché menu, sont traités dans un mortier avec peu d'eau distillée à $40^{\circ} \mathrm{C}$, puis la bouillie est versée quantitativement dans un matras taré de $250 \mathrm{~cm}^{3}$, 

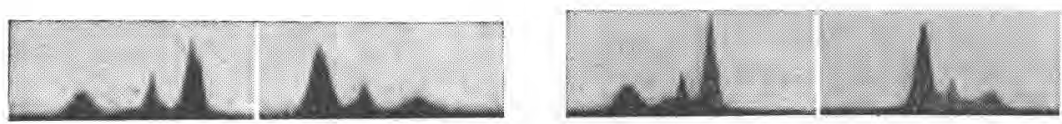

Fromage Italique
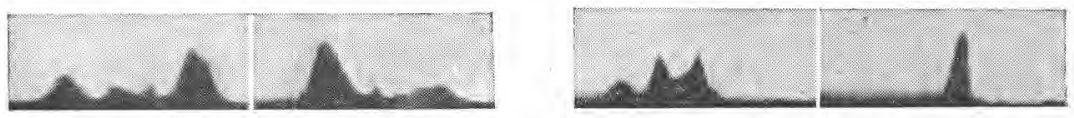

Fromage Gorgonzola
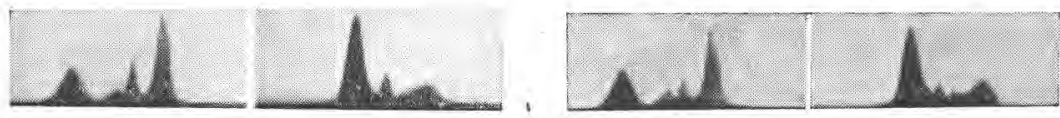

Fromage Mozzarella
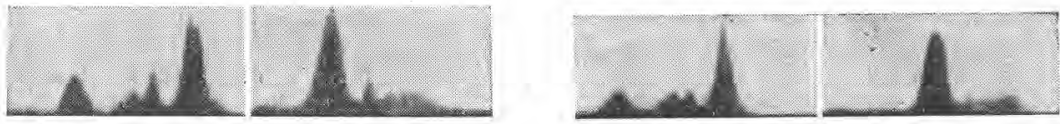

Fromage Provolone
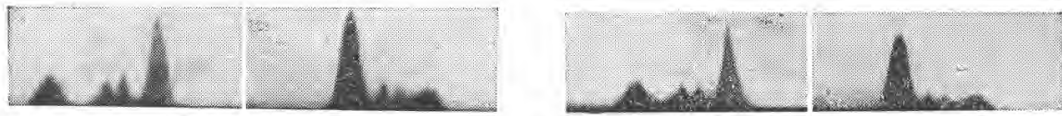

Fromage Fontina
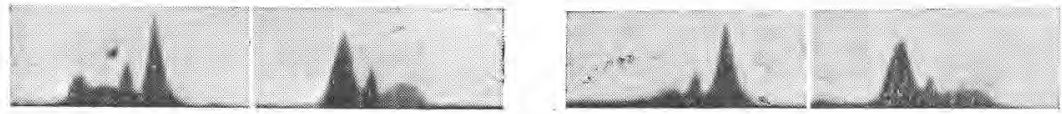

Fromage de brebis
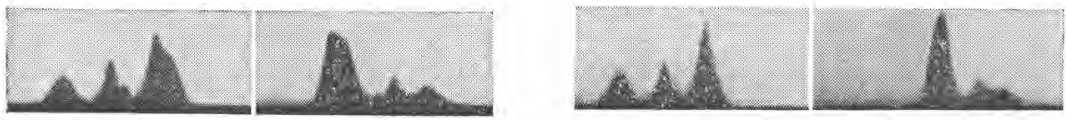

Fromage Parmesan 
auquel on ajoutera l'eau de lavage du mortier jusqu'au volume total. On laisse le tout, en agitant souvent, pendant 15 heures à température ambiante. Cette période de temps écoulée, on recueille le résidu par filtrage ou par centrifugation et on le délaie à $40^{\circ} \mathrm{C}$. en $40 \mathrm{~cm}^{3}$ de tampon. La solution est ensuite ultra-centrifugée et dialysée, d'après la méthode qu'on vient de décrire. La perte d'azote qu'on remarque pendant la dialyse est assez inférieure à celle qui a lieu avec le fromage entier; environ 10\%. Cela arrive parce que le fromage dans l'eau se dépouille de toutes les substances azotées solubles, dialysables en général, telles les amminoacides, les peptons, les protéoses simples, etc.

\section{Résultats obtenus}

Tout en reportant ci-après les électrophorétogrammes obtenus, on résume dans le tableau ci-dessous, les remarques relatives.

\begin{tabular}{|c|c|c|c|c|c|c|c|c|c|c|}
\hline \multirow{2}{*}{ Fromage } & \multicolumn{10}{|c|}{ Fractions caséiniques présentes dans la branche ascendante } \\
\hline & \multicolumn{5}{|c|}{ Fraction totale } & \multicolumn{5}{|c|}{ Fraction insoluble } \\
\hline Italique ..... & $\alpha_{1}$ & $\alpha_{2}$ & $\beta_{1}$ & $\beta_{2}$ & $\delta$ & - & $\alpha_{2}$ & $\beta_{1}$ & $\beta_{2}$ & $\delta$ \\
\hline Gorgonzola ... & $\alpha_{1}$ & $\alpha_{2}$ & $\beta_{1}$ & $\beta_{2}$ & $\delta$ & - & - & $\beta_{1}$ & $\beta_{2}$ & $\delta$ \\
\hline Mozzarella ........ & $\alpha_{1}$ & $\alpha_{2}$ & $\beta_{1}$ & $\beta_{2}$ & $\delta$ & - & $\alpha_{2}$ & $\beta_{1}$ & $\beta_{2}$ & $\delta$ \\
\hline Provolone ........ & $\alpha_{1}$ & $\alpha_{2}$ & $\beta_{1}$ & $\beta_{2}$ & $\delta$ & - & $\alpha_{2}$ & $\beta_{1}$ & $\beta_{2}$ & $\delta$ \\
\hline Fontine $\ldots \ldots \ldots$ & - & $\alpha$ & $\beta_{1}$ & $\beta_{2}$ & $\delta$ & & $\alpha$ & $\beta_{1}$ & $\beta_{2}$ & $\delta$ \\
\hline From. lait de brebis. & 一 & $\alpha$ & $\beta$ & - & $\delta$ & & $\alpha$ & $\beta$ & - & $\delta$ \\
\hline Parmesan ....... & $\alpha_{1}$ & $\alpha_{2}$ & $\beta_{1}$ & $\beta_{2}$ & $\delta$ & - & $\alpha_{2}$ & $\beta_{1}$ & $\beta_{2}$ & $\delta$ \\
\hline
\end{tabular}

D'après le relevé ci-dessus, on remarque que les fromages examinés ne contiennent pas le même nombre de fractions. La fraction $\alpha_{1}$ manque dans les deux fromages demi-cuits, Fontine et Fromage de lait de brebis; à ce dernier manque aussi la fraction $\beta_{1}$. La fraction $\alpha_{1}$ paraît très soluble en eau, comme le montre son absence constante dans la fraction insoluble. Solubilité qui semble être caractère particulier, cette fraction étant soluble même en fromages frais. Le fromage de lait de brebis s'écarte quelque peu des autres. fromages produits avec du lait de vache, mais cela se comprend aisément, le lait de brebis lui-même ayant un comportement électrophorétique différent du lait de vache.

\section{Discussion des résultats}

L'examen des électrophorétogrammes rapportés confirme que chaque fromage présente une physionomie électrophorétique 
particulière, laquelle dépend probablement de la technologie et du degré de maturation. Comparant les fromages soumis à l'expérience par groupes semblables, on peut remarquer dans les fromages crus, une fraction $\alpha_{1}$ qui manque dans les fromages demi-cuits mais non dans les fromages cuits, et une certaine prépondérance quantitative de la fraction $\beta_{1}$ sur la $\beta_{2}$, prépondérance qui se réduit à quantité égale pour les demi-cuits (Fontine) et à minorité dans le cuit, où la $\beta_{2}$ est en quantité supérieure à la $\beta_{1}$.

Entre fromages technologiquement semblables comme la "Mozzarella " et le "Provolone", on peut remarquer dans la fraction totale une ressemblance considérable, ressemblance qui n'existe évidemment plus dans les respectives fractions insolubles, le "Provolone" ayant subi une période de maturation bien plus longue et montrant, par rapport à la "Mozzarella» un degré plus élevé de protéolyse.

Les fractions auxquelles la maturation porte le plus d'atteinte sont particulièrement la $\alpha_{1}$ et la $\beta_{1}$.

Dans le relevé ci-dessous, on résume les fractions qui paraissent le plus solubilisées dans chaque fromage.

\begin{tabular}{|c|c|c|c|c|c|c|}
\hline Fromage & Frac & $18 \mathrm{~d}$ & brar & e as & lante & $\begin{array}{c}\text { Protéoses } \\
\text { inertes }\end{array}$ \\
\hline $\begin{array}{l}\text { Italique } \ldots \ldots \ldots \ldots \\
\text { Gorgonzola } \ldots \ldots \ldots \ldots \\
\text { Mozzarella } \ldots \ldots \ldots \\
\text { Provolone } \ldots \ldots \ldots \\
\text { Fontine } \ldots \ldots \ldots \\
\text { From. de lait de brebis } \ldots \\
\text { Parmesan } \ldots \ldots\end{array}$ & $\begin{array}{l}\alpha_{1} \\
\alpha_{1} \\
\alpha_{1} \\
\alpha_{1} \\
- \\
- \\
-\end{array}$ & $\alpha_{2}$ & $\begin{array}{l}\beta_{1} \\
\beta_{1} \\
\beta_{1} \\
\beta \\
\beta_{1}\end{array}$ & $\begin{array}{l}\beta_{2} \\
\beta_{2} \\
- \\
\beta_{2}\end{array}$ & $x$ & $\begin{array}{l}\text { augmentent } \\
\text { diminuent } \\
\text { augmentent } \\
\text { diminuent } \\
\text { invariés } \\
\text { diminuent } \\
\text { invariés }\end{array}$ \\
\hline
\end{tabular}

Les protéoses qui constituent les limites $\delta$ et $\varepsilon$ diminuent dans les fromages plus mûrs, ce qui atteste en eux un degré de protéolyse plus avancé. Dans les fromages crus comme l'Italique et le Gorgonzola, paraissent atteintes d'une façon prépondérante, les fractions $\alpha$. Dans les fromages à pâte filée, les fractions solubilisées sont principalement $\alpha_{1}$ et. $\beta_{1}$; dans les fromages demi-cuits, on remarque une $\beta$ solubilisation et dans le fromage de lait de brebis, la disparition de fraction pas exactement définies et identifiées, qui peuvent être rapportées au lait de brebis.

En ce qui concerne le fromage Parmesan de longue maturation, toutes les fractions paraissent plus ou moins atteintes, quoique d'autres études aient fait ressortir que les modifications les plus importantes sont à la charge de la fraction $\beta_{1}$. 


\section{Résumé}

Puisque les fromages présentent avec l'électrophorèse une physionomie particulière, dépendant de la technologie et du degré de maturation, on a observé le comportement électrophorétique de sept fromages italiens de façon à en comprendre deux crus, l'Italique et le Gorgonzola ; deux à pâte filée, Mozzarella et Provolone ; deux demi-cuits, la Fontine et le Fromage de lait de brebis, et un cuit, le Parmesan.

Les échantillons ont été examinés intégralement et dans leur résidu insoluble dans l'eau, de façon à pouvoir observer quelles fractions caséiniques apparaissent solubilisées. L'appareil pour électrophorèse utilisé est un Hilger et Watts; avee système optique Schlieren Philpot pour l'absorption de l'ultra-violet; on a encore utilisé une ultracentrifuge préparatoire Spinco. Les observations, effectuées après 90 minutes d'électrophorèse, ont été faites à $p \mathrm{H} 8,6$ - force ionique 0,1 - avec un courant de $12 \mathrm{~mA}$. Les résultats obtenus confirment l'individualité électrophorétique des fromages. Dans les fromages crus, dans les fromages à pâte filée et dans le Parmesan apparaissent cinq fractions, quatre dans la Fontine; le Fromage de lait de brebis s'écarte assez remarquablement des autres, produits avec du lait de vache. Les fractions caséiniques les plus atteintes par la maturation sont le $\alpha_{1}$ et la $\beta_{1}$, la première particulièrement pour les fromages erus, la deuxième pour les fromages demi-cuits et à pâte filée.

(Institut expérimental de Zootechnie. Modène. Directeur : O.. Parisi.)

\section{BIBLIOGRAPHIE}

[1] T. StorgÅrds, B. Lindquist. Centr. Lab. Mjolkcentralen. Stockholm, 1952 .

[2] B. Lindquist, T. Storg̊̊rds, M. B. Goransonn. Centr. Lab. Mjolkcentralen. Stockholm. 1952.

[3] B. LINDQVIst, T. STORG̊̊RDS. Milchwissenschaft, 12, 462. 1958.

[4] T. Tsugo, K. Yamauchi. Atti del XIV conv. intern. latte Roma, vol. II.

[5] S. Annibaldi. Il latte, 1, 25, 1958.

[6] T. Nakanishi, F. Tokita. Milchwissenschaft, 1, 9, 1958.

[7] Th. Payens. Neder. Melken Zuiveltijdschr, 12, 99, 1958.

[8] O. PARIsI. Il formaggio Grana. Soc. Ed. Modenese. Edit. IIIe. 1958.

[9] E. Carbone. Il formaggio Fontina Ed. La Moderna. Lodi. 1955.

[10] A. CAmpus. Il pecorino romano e sardo. Ed. Arte Stampa. Roma, 1936.

[11] E. Savini. Il Gorgonzola. Ed. Arte Stampa. Roma. 1936.

[12] C. Des Bo. Il Bel Paese. Ed. Arte Stampa. Roma. 1936.

[13] G. Rossi. Manuale di tecnologia casearia. Ed. Agricole. Bologna. 1958.

[14] E. Savini. Il formaggio provolane. Ist. Caseificio. Lodi.

[15] Hs. Nitschmann, W. Lehmann. Experientia, III, 157, 1947.

[16] E. Cherbuliez, P. Baudet. Helv. Chim. Acta, 33, 1673, 1950. 